\title{
Scanning Transmission Electron Microscopy (STEM) Tomography of Layer-by- Layer PAH/PSS-Au Nanocomposite Structures
}

\author{
Nabil D. Bassim*, Andrew Herzing**, Walter J. Dressick*, Kathryn Wahl*, Dmitri Y. Petrovykh*, \\ Kenan P. Fears*, Rhonda M. Stroud*, Thomas D. Clark* \\ * U.S. Naval Research Laboratory, Washington, DC, 20375 \\ ** Materials Measurement Laboratory, National Institute of Standards and Technology, \\ Gaithersburg, MD, 20899
}

Nanocomposite multilayered thin film architectures comprising polyelectrolytes and $\mathrm{Au}$ nanoparticles are an attractive platform for designing customized coatings for potential mechanical or sensing applications. We are studying coatings prepared via layer-by-layer (LbL) assembly of the oppositely-charged polyelectrolytes, polyallylamine hydrochloride (PAH) and sodium polystyrene sulfonate (PSS), deposited from various salt solutions $(\mu=1.00 \mathrm{M})$ to control film morphology, with the addition of regularly interspaced layers of $\sim 15-\mathrm{nm}$ Au-citrate nanoparticles following the method of Schmitt et al. [1]. Figure 1a and $1 \mathrm{~b}$ show that changing the salt counterion in the polyelectrolyte solutions from singly $\left(\mathrm{Cl}^{-}\right)$to doubly charged $\left(\mathrm{SO}_{4}{ }^{2-}\right)$ species alters not only the nanocomposite thickness and morphology, but also film uniformity and the Au nanoparticle packing and clustering. Figure 1c shows an AFM topographic image of the top surface of an LbL composite with $5 \mathrm{Au}$ nanoparticles layers $\left(2\left(\mathrm{Cl}^{-}\right) / 3 \mathrm{SO}_{4}{ }^{2-}\right)$ showing both large ripples in the films and finer-scale roughness.

While the cross-sectional TEM data allows us to inspect the distribution of Au particles within each layer, the regularity and spacing of the interparticle packing is inaccessible due to the projection nature of the technique. Similarly, the AFM measurements can tell us about the surface topology, while the internal structures remain hidden. In order to more fully understand these features, we have carried out STEM-based electron tomography in an aberration-corrected STEM. Slices extracted from the resulting electron tomogram were used to inspect the in-plane particle distributions, which were found to be highly dependent on the valency of the ions in solution during the deposition step. In general, the surface roughness, layer spacing and in-plane Au particle packing increased for samples deposited from polyelectrolyte solutions with the divalent anion $\mathrm{SO}_{4}{ }^{2-}$, relative to solutions with monovalent anions $\left(\mathrm{Cl}^{-}\right)$. Figure 2 shows a cross-sectional image of polymer/Au stacks prepared with both ion deposition conditions within one sample with color-coded arrows pointing to X-Z plane slices extracted from the tomogram from successive layers of the $\mathrm{Cl}^{-}$and $\mathrm{SO}_{4}{ }^{2-}$ counter ion specimen. The ordering present in the $\mathrm{Cl}^{-}$layers differs markedly from that in the $\mathrm{SO}_{4}{ }^{2-}$ layers. While the $\mathrm{Au}$ particles in the $\mathrm{Cl}^{-}$layers are evenly dispersed and well-defined, the particles in the $\mathrm{SO}_{4}{ }^{2-}$ layers are far more irregular and in many cases appear to have agglomerated into larger structures. Finally, the topography of each layer can be discreetly sampled on a particle by particle basis by inspecting the slices from each layer. In all cases, the $\mathrm{SO}_{4}{ }^{2-}$ layers were rougher and, in one slice, even exhibited a large semi-circular vertical perturbation. We posit that all three changes (roughness, polyelectrolyte layer thickness, and Au particle layer thickness/disorder) result from the ability of sulfate, but not chloride, to electrostatically bridge and self-assemble PAH polyelectrolyte chains in solution and transfer them as semi-organized structures to the multilayer surface during the deposition process.

[1] J. Schmitt, G. Decher, et al., Adv. Mater. 9, 61 (1997)

[2] This research was supported by the Naval Research Laboratory's Nanoscience Institute. 


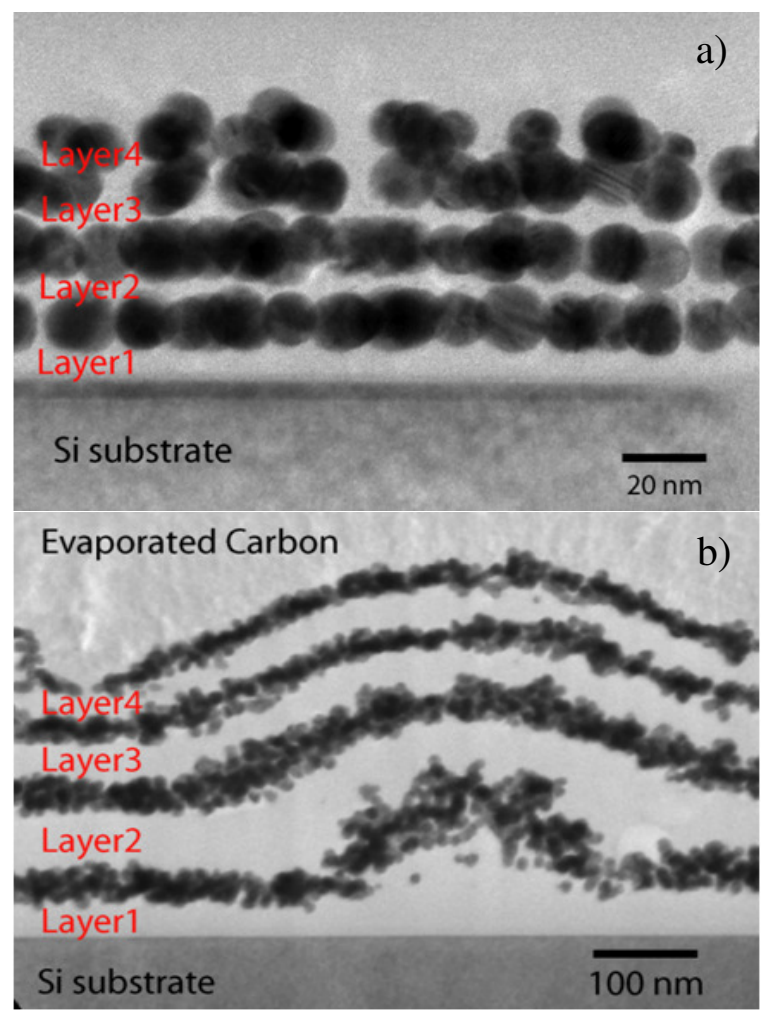

c)

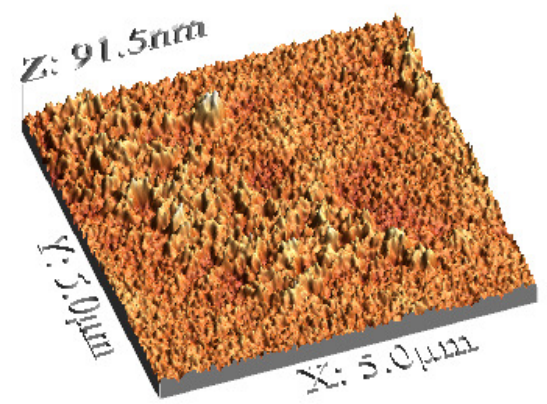

Figure 1: TEM BF cross-sectional images of 4-Au/4 PAH/PSS bilayer stacks stabilized by a) $\mathrm{Cl}^{-}$and b) $\mathrm{SO}_{4}{ }^{2-}$ counter ions. c) AFM topograph showing rough polymer surface layer in samples prepared with $\mathrm{SO}_{4}{ }^{2-}$ valence.
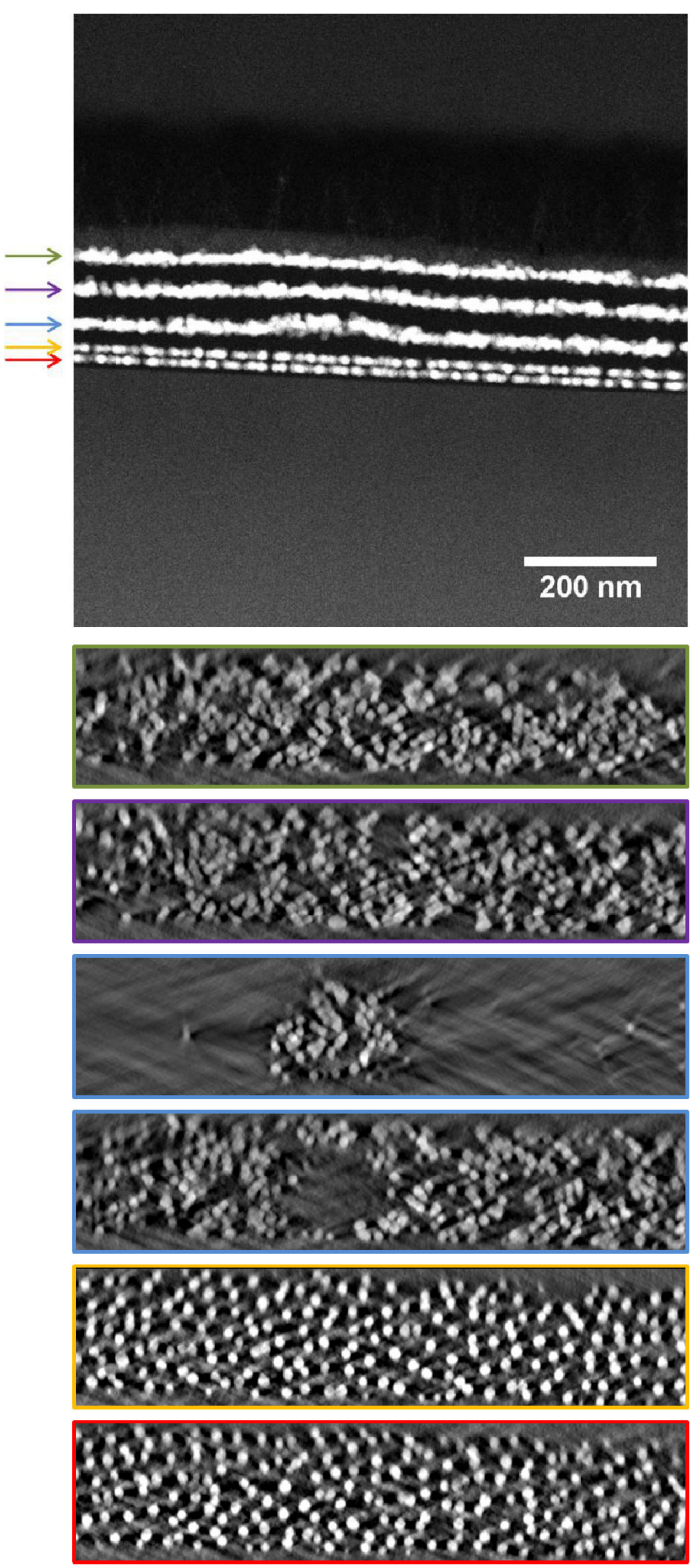

Figure 2: Cross-sectional STEMHAADF image of PSS/PAH-Au nanocomposite with both types of $\mathrm{LbL}$ morphology $\left(\mathrm{Cl}^{-}\right.$-stabilized, bottom 2 layers, $\mathrm{SO}_{4}{ }^{2-}$, top 3 layers) with colorcoded $\mathrm{X}-\mathrm{Z}$ slices based on the STEM tomographic reconstruction. Particle spacing is more even and sparse in $\mathrm{Cl}^{-}$stabilized layers than $\mathrm{SO}_{4}{ }^{2-}$ stabilized layers. 